\title{
Study on the Characteristic of External Conformal Turbine for Underwater Moving Body Measurement
}

\author{
Jiangfeng Tu, Shaojie Ma, He Zhang \\ Nanjing University of Science and Technology \\ Nanjing, China \\ 1192796462@qq.com; 438729185@qq.com; hezhangz@njust.edu.cn
}

\begin{abstract}
The external turbine mechanism is a new sensor that can be used for independent velocity measurement of underwater moving body. In order to study the relationship between the flow rate and the speed of the turbine, firstly, the structure and working principle of the turbine mechanism are given. Then, based on the turbine rotation equation, the dynamic CFD simulation method and the realization process are provided. The water tunnel experiment is carried out to verify the performance of the turbine speed measurement. At the same time, the effect of cavitation and small angle of attack on the performance of turbine speed measurement are also studied. Research results show that, there is a good linear relationship between flow rate and rotational speed. Tests show that the cavitation can lead to the decline of the turbine speed, which leads to the failure of the turbine speed measurement. At the angle of attack, the output characteristic of the turbine speed measurement mechanism is changed.
\end{abstract}

Keywords: Underwater moving body; independent velocity measurement; External conformal turbine; Water tunnel test.

\section{Introduction}

There are some special problems in the velocity measurement of the underwater moving object compared with the atmospheric environment. For example, the water has a much larger viscous damping than the air, and moving objects in the water has a larger resistance; Water has the electrical conductivity, the appropriate insulation, the shield and the waterproof technology must be taken[1]. Sky target velocity, sound velocity measurement target, radar, GPS velocity determination, microwave interferometry and high speed photography method cannot be well adapt to the underwater environment [2]. And the use of a turbine or turbine flowmeter to measure the fluid parameters, has been the main means of fluid testing, and a lot of relevant research has been carried out. Pankaj K. Jha [3] studied blade Load Unsteadiness and Turbulence Statistics in an Actuator-Line Computed Turbine-Turbine Interaction Problem. Jianliang Zhao [4] studied the Viscosity Impact of Turbine Flowmeter's Performance by experiment and numerical simulation. Zoheir Saboohip [5] developed a model for prediction of helical turbine flowmeter performance using CFD.

An external turbine measurement mechanism was designed in this paper, making use of a structure similar to conventional turbine measuring device (such as a turbine flowmeter) and considering the special characteristics of the underwater moving body. By modeling and designing simulation program, the characteristics of the sensor were analyzed in detail.

\section{Sensor Structure and Working Principle}

\subsection{Sensor structure}

In order to reduce the influence of the external turbine on the performance of the surrounding flow field, the shape of the turbine is consistent with the moving body. The basic principle of measuring the velocity of the external conformal turbine mechanism is as shown in Fig.1. 


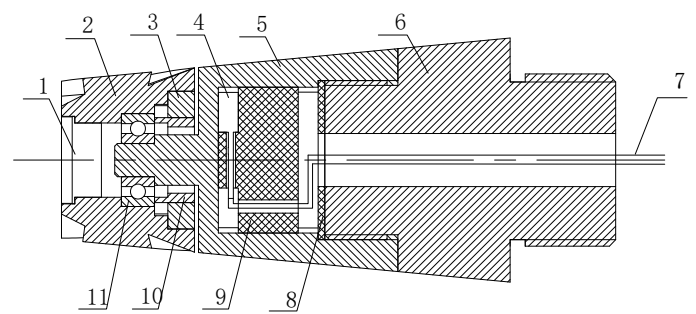

Fig.1: Schematic diagram of turbine mechanism. (1- End cap; 2- turbine; 3- Small magnet; 4- Hall Device; 5- Connecting piece; 6Moving body; 7- wire; 8- Sealing ring; 9- Hall Device holder; 10- Fastening ring; 11- Bearing).

Working principle: when the moving body is moving in the underwater, the turbine is driven by the force of the fluid to rotate. A small magnet embedded in a turbine generates periodic variation of the magnetic field, Hall device tests the change of magnetic field to measure the speed of the turbine, and there is a certain function relation between the speed and the speed of the moving body. At the same time, the speed signal is input to the MCU. MCU can get the velocity information of the moving body, and can also calculate the distance, acceleration and other information of the moving body.

When the movement speed is higher, the measuring range is beyond the traditional turbine flowmeter. The turbine speed measuring mechanism is directly mounted on the head of the moving body, and is moving along with the moving body, and has a bigger measuring range. Therefore, it is necessary to analyze the characteristics of the turbine mechanism and flow field analysis.

\subsection{Parameters of the sensor structure}

It is necessary to give the sensor structure parameters for further analyze the influence of structure on sensor characteristic. Parameter diagrams of the turbine are shown as Fig.2, with important dimensions and curves marked.

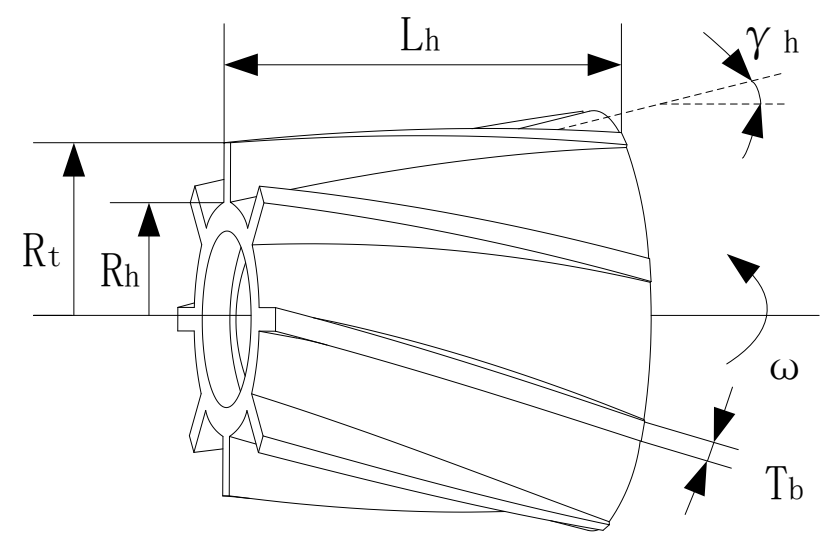

Fig. 2: External turbine configuration and main parameters.

The main geometric parameters of the external turbine : Minimum hub radius $R_{h}$ 、Minimum tip radius $R_{t}$, leaf angle ${ }^{\gamma_{t}}$, Hub length $L_{h}$, Hub surface dip angle $\gamma_{h}$, leaf number $N$, Blade guide $L_{d}$ and thickness $t_{b} . \Delta \omega$

\section{Modeling and Simulation}

\subsection{Flow field}

In order to study the characteristics of the turbine mechanism under the condition of complicated and changeable water environment, a computational fluid dynamics model is established to analyze the characteristics of the sensor. 


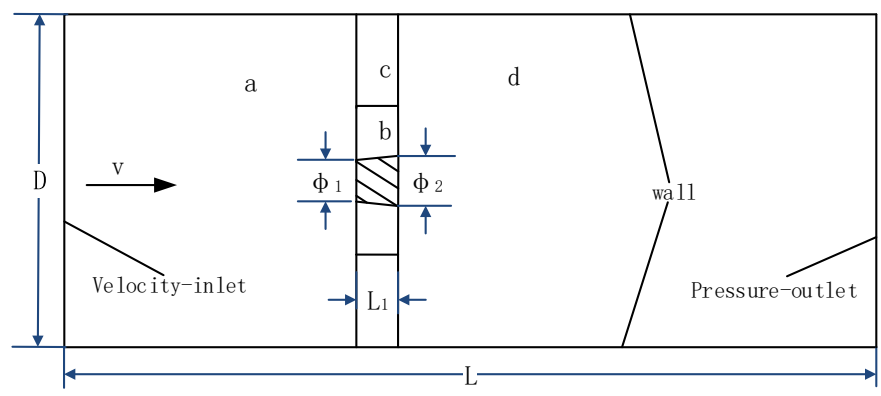

Fig. 3: Flow field division.

The whole flow field can be divided into several areas as shown in Fig.3, a and $\mathrm{d}$ are the turbine before and after the areas, $\mathrm{b}$ and $\mathrm{c}$ is the turbine areas, where $\mathrm{d}$ for the rotating area.

In order to ensure the free wall effect, the diameter of the flow field is $D=20 \phi_{2}$. In addition, in order to ensure that the simulation is not affected by the back flow, $\mathrm{L}=30 \mathrm{DH}$.

\subsection{Meshing}

O-block meshing method is used to mesh the flow field, meshing scheme is shown as Fig.4. Single / double domain partitioning based on structured grids, the characteristic of this kind is relatively simple in the flow field, and the whole flow field is integrated into the grid, and the grid does not change with time. The advantages of the unified structure of the whole flow field is that the accuracy of the flow field is higher, and the solution is faster. Especially suitable for the higher requirement of turbulence model. The meshing method can be used to study the unsteady characteristics, such as the unsteady characteristics of turbulent flow or cavitation itself, and the dynamic characteristics of the turbine mechanism itself.
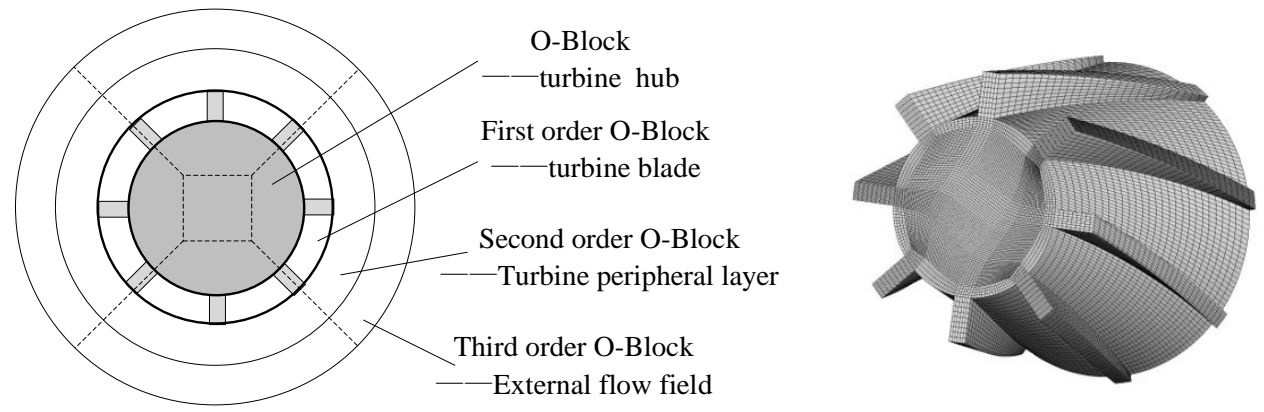

A. Flow field meshing

B. Turbine surface meshing

Fig. 4: Flow field and Turbine surface mesh generation.

Grid quality directly determine the calculation's convergence, efficiency and accuracy, we should make a reasonable meshing according to the distribution characteristics of the physical parameters in the flow field. By using the boundary layer and the spacing size function of control grid, the boundary layer grid is encrypted and done some corresponding adjustment. The turbine head of the turbine rotating area and the near wall are encrypted in the height direction perpendicular to the wall surface, and the other areas are relatively sparse. On the basis of guaranteeing the quality of the grid, the amount of computation and the total number of meshes is controlled, and the turbine surface meshing is shown in Fig.4 B.

\subsection{Solving process}

According to the turbine dynamic rotation equation [6] [7] [8] and the correlation analysis results of the above modeling method, the dynamic rotation of the turbine can be simulated by UDF program. The program flow chart is shown as Fig.5 : 


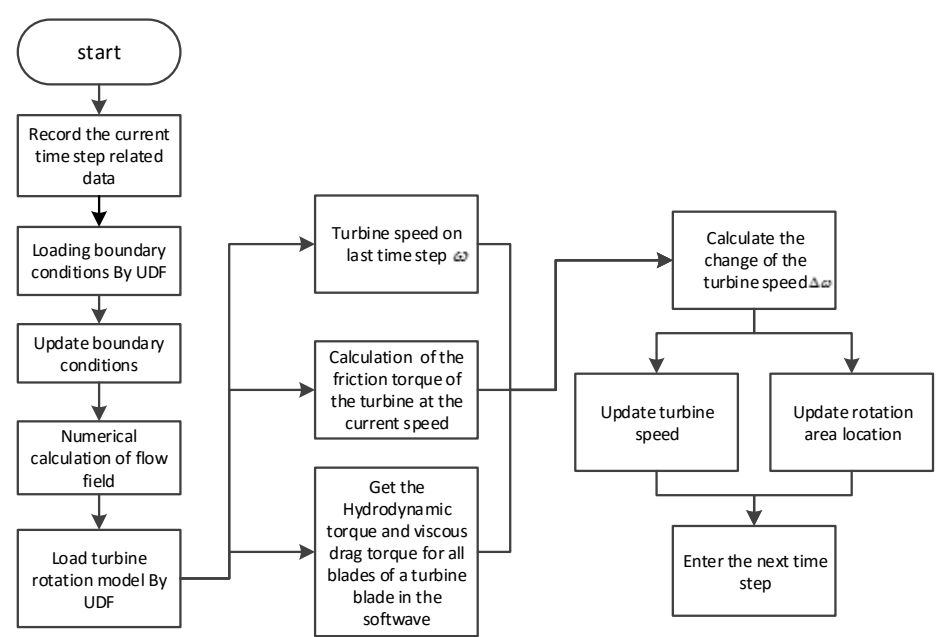

Fig. 5: Turbine dynamic CFD implementation process.

Dynamic CFD simulation of turbine under dynamic flow conditions (for example the ideal order step signal, acceleration and deceleration) is shown in the figure above, where the speed of inlet and outlet to simulate the pressure changed by the UDF programming. The steady and unsteady characteristics of the turbine are analyzed by the above method, and the corresponding critical fluid parameters are monitored.

\section{Water tunnel test}

In order to study further the sensor characteristics of turbine, the water tunnel experiment was carried out, Schematic of the test system is shown in Fig.6. Prototypes and water tunnel equipment were shown in Fig.7. Experiments were carried out in the K15 cavitation tunnel. The cross-section of tunnel experimental section is a $1 \mathrm{~m}^{*} 1 \mathrm{~m}$ square, with flow speed changed from $0 \mathrm{~m} / \mathrm{s}$ to $12 \mathrm{~m} / \mathrm{s}$ continuously and adjustable pressure. The test results and calculation result were contrasted in Fig. 8, with the pressure of $1 \mathrm{~atm}$, and the flow speed was from $5 \mathrm{~m} / \mathrm{s}$ to $10 \mathrm{~m} / \mathrm{s}$.

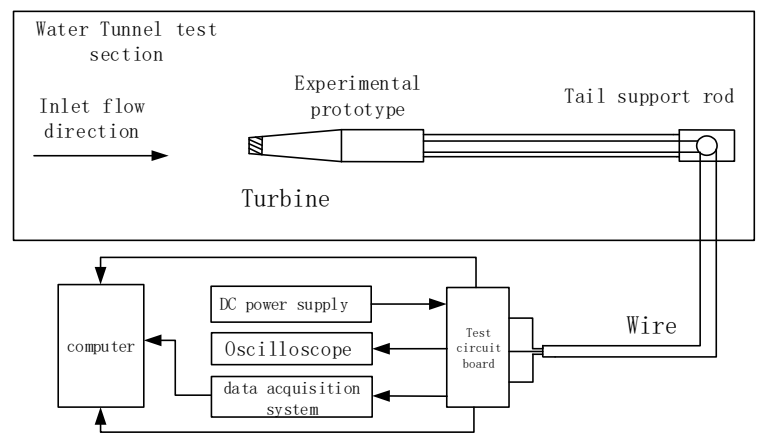

Fig. 6: Cavitation in water tunnel test schematic diagram.

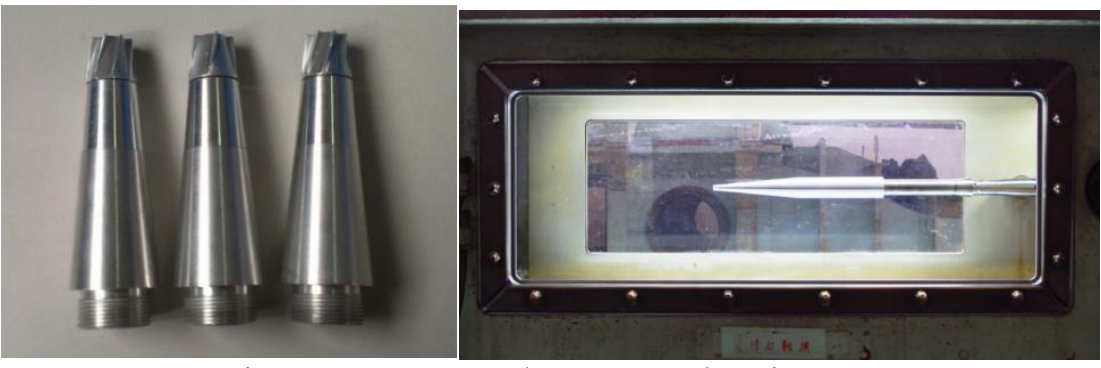

Fig. 7: Prototypes and water tunnel equipment. 


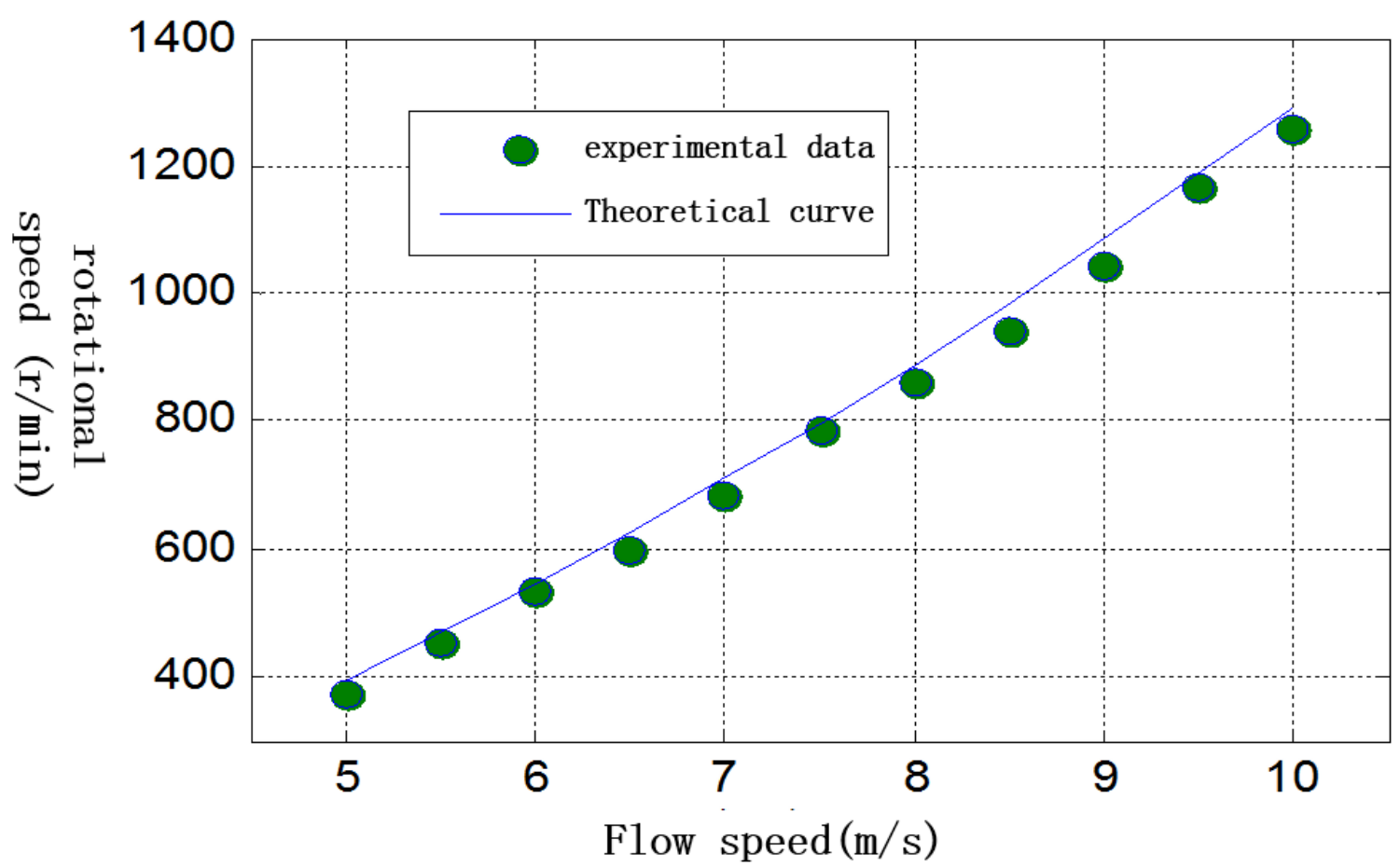

Fig. 8: Date of test results and calculation result.

As can be seen from the figure, the turbine speed and flow rate in the experimental section has a good linearity, based on the least squares method for linear fitting, nonlinear degree of 0.995 . The data calculated by the theory is in good agreement with the experimental results. At each test point, the maximum error of theoretical calculation is about $4.9 \%$.

In order to study the influence of cavitation on the rotation of the turbine and determine the incipient cavitation number, the cavitation number is changed by unchanging flow rate and Pressure adjustment. The turbine speed data and the cavitation of the turbine surface are recorded under different cavitation numbers.

At the speed of $10 \mathrm{~m} / \mathrm{s}$, the cavitation number is reduced by reducing pressure. The cavitation of different cavitation numbers is shown in Fig.9. According to the measured data, the incipient cavitation number is 0.8 .

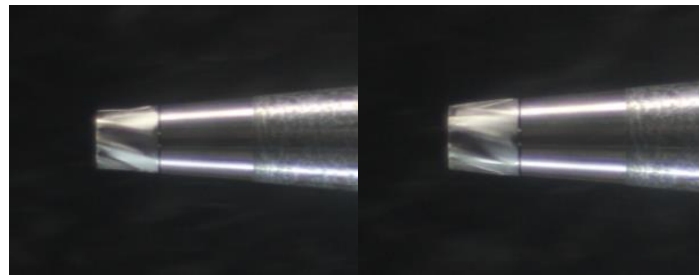

(a) cavitation number (b) cavitation number is 1.0

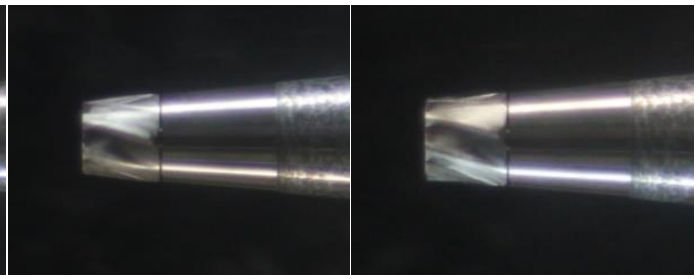

(c) cavitation number (d) cavitation number is 0.8 is 0.7

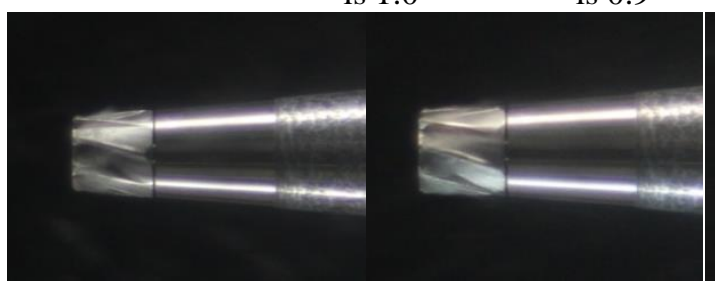

(e) cavitation number (f) cavitation number is 0.6 is 0.5

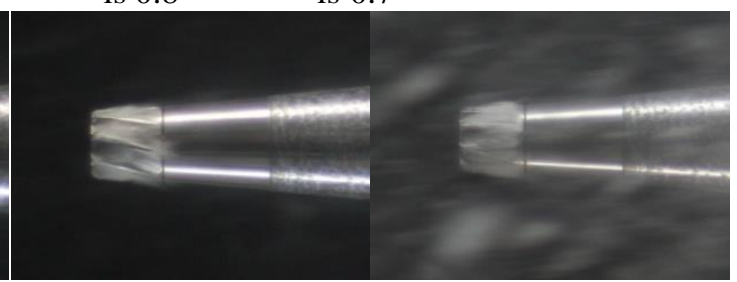

(g) cavitation number $(\mathrm{h})$ cavitation number is 0.4 is 0.33

Fig. 9: Photos of different cavitation.

The influence of cavitation number on the turbine speed is shown in Fig.10. 


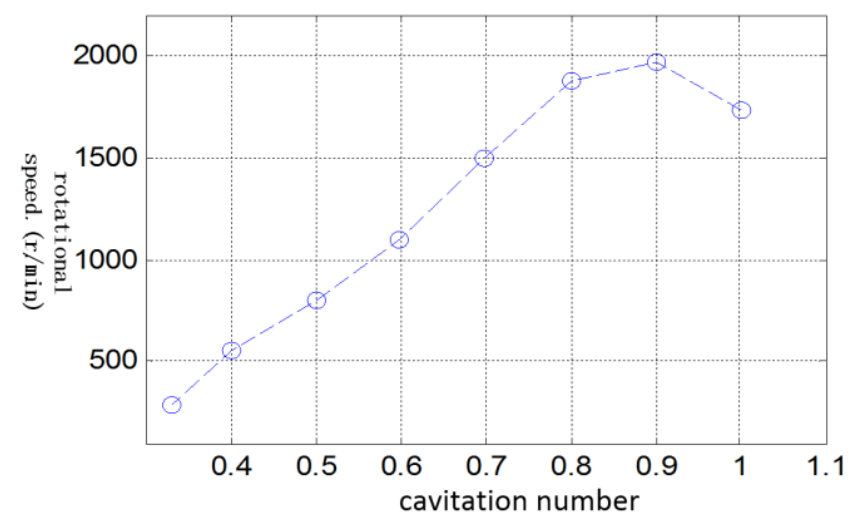

Fig. 10: Effect of cavitation on turbine speed.

The incipient cavitation number is 0.8 , and the smaller the number of cavitation for the more serious cavitation. Can be seen from the figure, after the occurrence of cavitation, with the reduction of the number of cavitation, the turbine speed is also reduced. But before the cavitation, the turbine speed will increase with the decrease of the number of cavitation. Under a certain velocity condition, the turbine has the maximum rotation speed when the cavitation is about to happen.

At the same time, the cavitation of turbine surface at different velocities was calculated based on the Sauer and Schnerr cavitation model. The gas phase volume fraction is calculated by the cavitation model at $30 \mathrm{~m} / \mathrm{s}$ (cavitation number is 0.6 ), $50 \mathrm{~m} / \mathrm{s}$ (cavitation number is 0.2 ) and $80 \mathrm{~m} / \mathrm{s}$ (cavitation number is 0.08 ) velocity. The results are shown in Fig. 11.
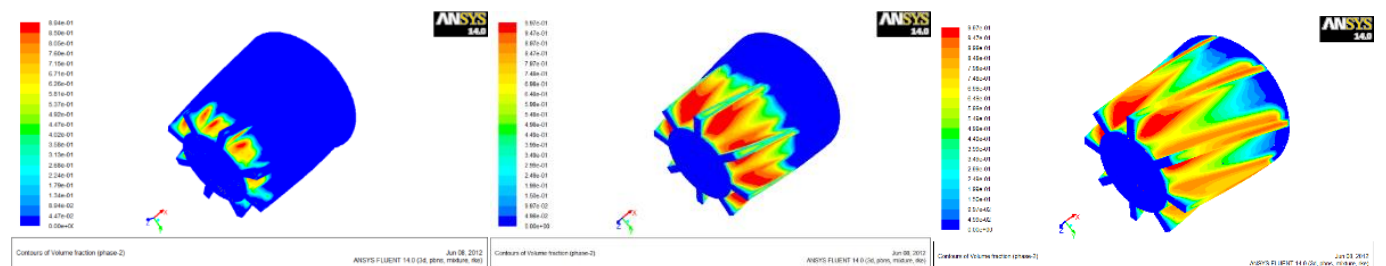

(a) cavitation number is 0.6 (b) cavitation number is 0.2 (c) cavitation number is 0.08

Fig. 11: Gas volume fraction of the surface of the turbine under different cavitation numbers.

In order to obtain the rotational speed of the turbine with small angle of attack, the measurement experiment of the turbine speed is carried out under the condition of small attack angle. Based on the experimental data, the corresponding curves are drawn as shown in Fig. 12.

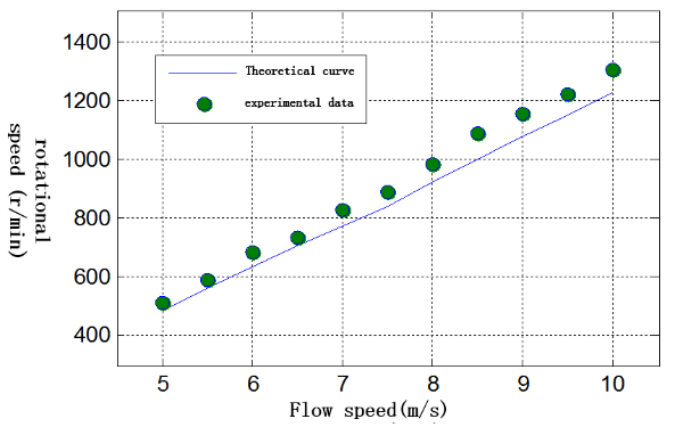

(a) Comparison of theoretical curves and experimental data

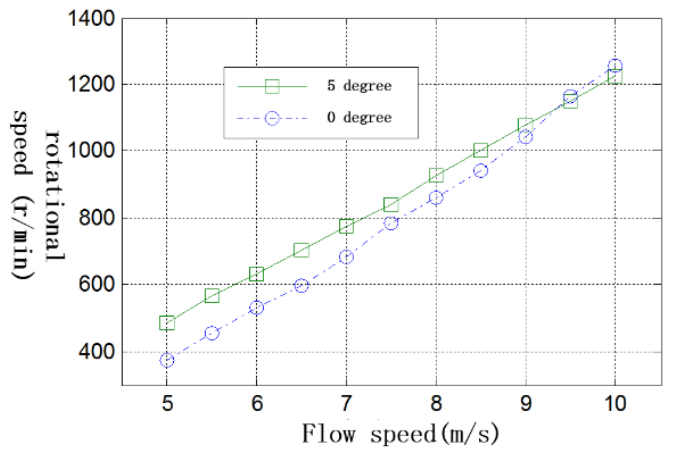

(b) Data comparison between 0 degree and 5 degree angle of attack

Fig.12: The test data under the condition of small angle of attack 
Can be seen from the figure above, 5 degree angle of attack, the theoretical calculated value is relatively smaller than the measured value, the maximum error is about $-6.6 \%$. 5 degree angle of attack of the turbine speed is bigger than the 0 degree angle of attack at low flow rate. At high flow rate, on the contrary, it is smaller.

\section{Conclusion}

The turbine speed measuring device is installed on the head of the moving body, and is moving along with the moving body, and has a range of velocity larger than that of the traditional turbine flowmeter. The data calculated by the theory is in good agreement with the experimental results. Using the least square method to fit the experimental data, there is a good linear relationship between flow rate and rotational speed. Tests show that the cavitation can lead to the decline of the turbine speed, which leads to the failure of the turbine speed measurement. At the angle of attack, the output characteristic of the turbine speed measurement mechanism is changed.

\section{Acknowledgements}

The authors gratefully acknowledge the support of National Natural Science Foundation of China (NO 51275248).

\section{References}

[1] Z. Wang, Y. Zhu, C. Wang, and D, Kong, "Discussion on measuring method of dynamic velocity of moving body in water [J]," Chinese Journal of Scientific Instrument, vol. 23, no. 3, pp. 20-21, 2002.

[2] P. Li, "Study on measuring technique of underwater high speed moving body [D]," Nanjing: Nanjing University of Science \& Technology, 2009.

[3] P. K. Jha and S. Schmitz, "Blade Load Unsteadiness and Turbulence Statistics in an Actuator-Line Computed TurbineTurbine Interaction Problem [J]," Journal of Solar Energy Engineering, vol. 138, pp. 031002-1, 2016.

[4] J. Zhao, L. Sun, T. Zhang, and L. Cao, "Experiment and Numerical Simulation Study on the Viscosity Impact of Turbine Flowmeter's Performance [J]," Advanced Materials Research, vol. 301-303, pp. 1283-1288, 2011.

[5] Z. Saboohi, S. Sorkhkhah, and H. Shakeri, "Developing a model for prediction of helical turbine flowmeter performance using CFD [J]," Flow Measurement and Instrumentation, vol. 42, pp. 47-57, 2015.

[6] S. Dezhang, Z. He, and L. Haojie, "Autonomous velocity measurement method for small underwater high velocity moving body," Chinese Journal of Scientific Instrument, vo1. 33, no. 12, 2012.

[7] P. Boltryk, M. Hill, A. Keary, et al., "An ultrasonic transducer array for velocity measurement in underwater vehicles [J]," Ultrasonics, vol. 42, pp. 473-478, 2004.

[8] G. H. Yan, Z. F. Chen, and J. C. Sun, "Using a linear array to estimate the velocity of underwater moving targets [J]," Journal of Marine Science and Application, vol. 4, no. 8, pp. 343-347, 2009. 